\title{
Frecuencia y características clinicopatológicas de mujeres obesas con cáncer de mama del Departamento de Oncología del Hospital de Clínicas
}

\author{
$*$ Justino Toledo $^{1}$ (iD) $* *$ Rita Denis $^{1}$ (i),$* * *$ Ignacio Ortiz Galeano $^{1}$ (D) \\ ${ }^{1}$ Universidad Nacional de Asunción, Facultad de Ciencias Médicas, Hospital de Clínicas. San \\ Lorenzo, Paraguay
}

Cómo referenciar este artículo/

How to reference this article:
Toledo J, Denis R, Ortiz Galeano I. Frecuencia y características clinicopatológicas de mujeres obesas con cáncer de mama del Departamento de Oncología del Hospital de Clínicas.Mem. Inst. Investig. Cienc. Salud. 2021; 19(1): 58-63

\section{RE S U M E N}

Ante el incremento de la obesidad en los últimos años, se observa un aumento no solo de factores de riesgo cardiovasculares, sino también de la incidencia de cáncer debido a su conocida participación en procesos de oncogénesis. El objetivo del estudio fue determinar la frecuencia y las características clinicopatológicas de las mujeres obesas con diagnóstico de cáncer de mama del servicio de Oncología del Hospital de Clínicas. Diseño observacional, descriptivo, retrospectivo de corte transversal, que incluyó a pacientes con diagnóstico de cáncer de mama del Servicio de Oncología del Hospital de Clínicas desde enero de 2017 a junio de 2019. Se determinaron las variables demográficas, índice de masa corporal, subtipo histológico, presencia de receptores hormonales y sobreexpresión del oncogén HER 2neu. Se analizaron 144 sujetos, la media de edad fue $52 \pm 12,2$ años, la procedencia más frecuente fue de ciudades del departamento central (54\%). El $40,2 \%$ presentó obesidad y $36,8 \%$ sobrepeso al momento del diagnóstico. El subtipo histológico más frecuente hallado en las mujeres con sobrepeso y obesidad fue el carcinoma ductal $(81,9 \%)$, presentaron receptores hormonales positivos a la inmunohistoquímica el $78,3 \%$ y sobreexpresión del oncogén HER 2 neu en $11,7 \%$. La frecuencia de obesidad en mujeres con diagnóstico de cáncer de mama del servicio de Oncología del Hospital de Clínicas fue elevada. Las características anatomopatológicas más frecuente fue el carcinoma ductal infiltrante, los datos biológicos muestran a los receptores de estrógenos y/o progesterona en mayor medida y sobreexpresión del oncogén el HER 2 neu en la menor parte de los casos.

Palabras clave: cáncer de mama, sobrepeso, obesidad.

\section{Frequency and clinicopathological characteristics of obese women with breast cancer in the Oncology Department of the Hospital de Clínicas}

\begin{abstract}
Given the increase in obesity in recent years, an increase is observed not only in cardiovascular risk factors, but also in the incidence of cancer due to its known participation in oncogenesis processes. The objective of the study was to determine the frequency and clinicopathological characteristics of obese women diagnosed with breast cancer in the Oncology service of the Hospital de Clínicas. Observational, descriptive, retrospective cross-sectional design, which included patients with a
\end{abstract}


diagnosis of breast cancer from the Oncology Service of the Hospital de Clínicas from January 2017 to June 2019. Demographic variables, body mass index, histological subtype, presence of hormone receptors and overexpression of the HER 2neu oncogene were determined. One hundred forty four subjects were analyzed, the mean age was $52 \pm 12.2$ years, and the most frequent origin was from cities of the central department (54\%). Forty point two percent was obese and $36.8 \%$ was overweight at the time of diagnosis. The most frequent histological subtype found in overweight and obese women was ductal carcinoma $(81.9 \%)$, and $78.3 \%$ had positive hormone receptors to immunohistochemistry and overexpression of the HER 2 neu oncogene was found in $11.7 \%$. The frequency of obesity in women diagnosed with breast cancer in the Oncology service of the Hospital de Clínicas was high. The most frequent anatomopathological characteristics were infiltrating ductal carcinoma, biological data show estrogen and / or progesterone receptors to a greater extent and overexpression of the HER 2 neu oncogene in the smallest part of the cases.

Keywords: breast cancer, overweight, obesity.

\section{INTRODUCCIÓN}

Se conoce a la obesidad como la epidemia del siglo XXI ante su incremento exponencial en los últimos años como consecuencia los malos hábitos como la alimentación hipercalórica y/o rica en grasas y carbohidratos y el sedentarismo ${ }^{(1,2)}$. Bien se conocen sus efectos adversos como parte del síndrome metabólico o individualmente como factor independiente de riesgo cardiovascular ${ }^{(3)}$. De la misma manera se conoce su participación en la oncogénesis de varios procesos tumorales como en los de esófago estómago, colorrectal, tiroides, páncreas, mama, mieloma múltiple; así como empeoramiento del pronóstico en ciertos casos ${ }^{(4-6)}$. Desde hace dos décadas se observó que tanto la actividad física como el índice de masa corporal y circunferencias del cuerpo influían en la menor frecuencia de aparición de cáncer de mama sobre todo en mujeres posmenopáusicas ${ }^{(7-9)}$. Posteriores estudios sobre la fisiopatología de la obesidad lleva a entender los mecanismos moleculares que promueven la oncogénesis a través de un estado proinflamatorio desarrollado por el tejido adiposo mediante la liberación de citoquinas que promueven la angiogénesis y la proliferación celular en distintos órganos del cuerpo, sobre todo en las células de las glándulas mamarias $(7,8,10,11)$. Sin embargo, dicha asociación fue variable de acuerdo a la edad de los pacientes y de acuerdo a la expresión de receptores hormonales presentes en las células tumorales: así por ejemplo, la mayor prevalencia se vio en pacientes posmenopáusicas con expresión positiva de receptores de estrógenos; sin embargo dicha asociación fue inversa en pacientes premenopáusicas con baja expresión de receptores hormonales ${ }^{(12-14)}$; es decir que la obesidad en mujeres premenopáusicas se asocia con mayor riesgo de cáncer de mama con receptores hormonales negativos.

El objetivo del estudio fue determinar la frecuencia y las características clinicopatológicas de las mujeres obesas con diagnóstico de cáncer de mama del servicio de Oncología del Hospital de Clínicas.

\section{MATERIAL Y MÉTODO}

Se aplicó un diseño observacional, descriptivo, retrospectivo, de corte transversal.

La población de estudio estuvo constituida por mujeres con diagnóstico de cáncer de mama en seguimiento por el servicio de Oncología del Hospital de Clínicas en el período de enero 2017 a julio 2019.

Los criterios de inclusión fueron pacientes mayores de 18 años con diagnóstico de cáncer de mama confirmada por biopsia. Fueron excluidas 8 historias clínicas incompletas. Se utilizó un muestreo no probabilístico de casos consecutivos.

Para el reclutamiento se solicitó autorización a las autoridades del Hospital de Clínicas. Se realizó una revisión retrospectiva de las historias clínicas de los pacientes. Se estudiaron variables sociodemográficas (edad y procedencia: Asunción, departamento Central e interior del país), índice de masa corporal, estudio histológico (clasificados en tipo ductal, tipo lobulillar y otros), presencia de receptores 
hormonales, estrógenos, progesterona o por inmunohistoquímica), y presencia de sobreexpresión del oncogén HER 2 neu.

Para el cálculo de tamaño de muestra se utilizó el programa estadístico EpiDat 3. $1^{\circledR}$. Para una población finita de 144 pacientes con diagnóstico de cáncer de mama en el periodo de estudio, frecuencia esperada de $90 \%$ de sobrepeso y obesidad (15), para una precisión 1\%, IC 95\%, el tamaño mínimo a incluir fue de 139 sujetos.

Los datos fueron cargados en una hoja de cálculo de Excel $®$ y analizados utilizando el programa estadístico Epi Dat 3.1. Se utilizó estadística descriptiva, expresando las variables cualitativas en frecuencias absolutas y relativas porcentual, y las cuantitativas en medias y desviación estándar.

Aspectos éticos: El protocolo fue aceptado por el Comité de Ética en investigación de la Facultad de Ciencias Médicas de la Universidad Nacional de Asunción. El estudio se realizó siguiendo las normativas establecidas en la Declaración de Helsinki para investigaciones que involucran seres humanos.

\section{RESULTADOS}

Se incluyeron en el estudio a 144 mujeres con cáncer de mama entre 32 y 84 años (edad media \pm DE 52士12,2 años, 25 (17\%) pacientes eran de Asunción; 78 (54\%) de ciudades del departamento Central y $41(29 \%)$ del interior del país.

La media de pesos de las pacientes con cáncer de mama fue de $71,48 \pm 12,59 \mathrm{~kg}$, la media de talla fue de $1,59 \pm 0,07$ metros y la media del IMC fue de $28 \pm 4,98 \mathrm{~kg} / \mathrm{m}^{2}$. La frecuencia de obesidad es del $40,2 \%$ y del sobrepeso $36,8 \%$ (Tabla 1 ).

Tabla 1: Frecuencia de índice de masa corporal de mujeres con diagnóstico de cáncer de mama del Departamento de Oncología del Hospital de Clínicas

\begin{tabular}{lll}
\hline Clasificación según IMC & $\mathbf{n ~ ( 1 4 4 )}$ & \% \\
\hline Bajo peso & 1 & 0,7 \\
Peso normal & 32 & 22,2 \\
Sobrepeso & 53 & 36,8 \\
Obesidad grado 1 & 41 & 28,5 \\
Obesidad grado 2 & 15 & 10,4 \\
Obesidad grado 3 & 2 & 1,4 \\
\hline
\end{tabular}

IMC: Índice de masa corporal.

En la población estudiada, se encontró en la anatomía patológica 117 pacientes $(81,2 \%)$ con carcinoma ductal infiltrante (Figura 1$)$.

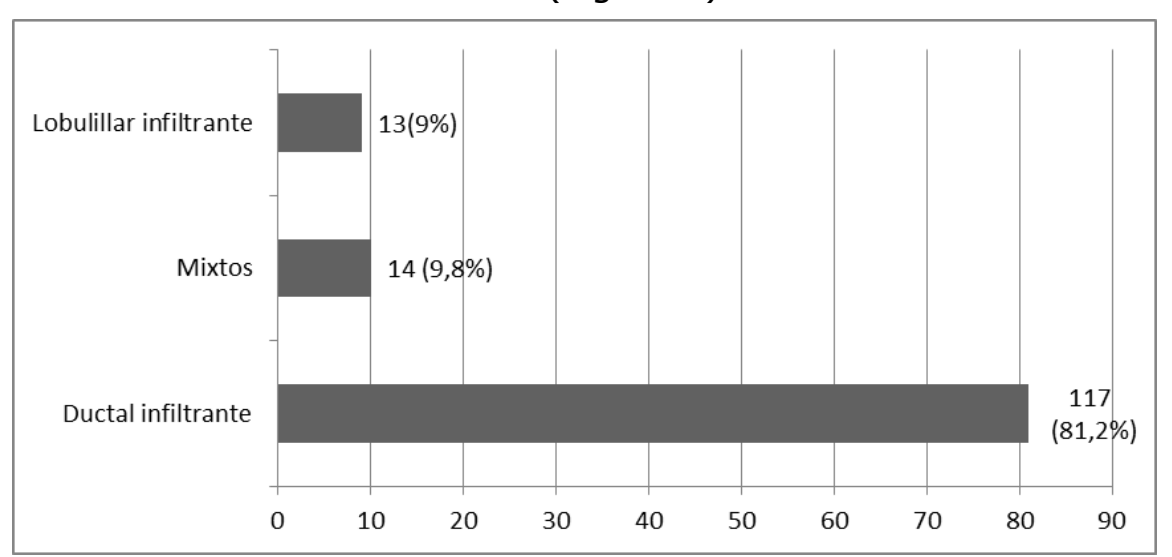

Figura 1: Tipo histológico en pacientes con cáncer de mama del Departamento de Oncología del Hospital de Clínicas $(n=144)$ 
En las 144 mujeres con cáncer de mama se encontró en los estudios inmunohistoquímica presencia de receptores hormonales estrógeno y/o progesterona en 116 pacientes ( $81 \%$ ) y sobreexpresión del oncogén HER 2 neu se observó en 15 pacientes $(10 \%)$.

En 111 pacientes $(77 \%)$ de las pacientes de cáncer de mama se encontró sobrepeso u obesidad en el momento del diagnóstico de la enfermedad, de las cuales $91(81,9 \%)$ tenían carcinoma de tipo ductal, 87 (78,3\%) con receptores hormonales positivos y $13(11,7 \%)$ con presencia de gen HER 2 neu (Tabla 2$)$.

Tabla 2: Características histopatológicas e inmunohistoquímicas en mujeres con sobrepeso u obesidad con cáncer de mama del Departamento de Oncología del Hospital de Clínicas $(n=144)$

\begin{tabular}{lcc}
\hline & $\begin{array}{c}\text { Sobrepeso u obesidad } \\
(\mathbf{n = 1 1 1})\end{array}$ & $\begin{array}{c}\text { Normopeso } \\
(\mathbf{n = 3 3})\end{array}$ \\
\hline $\begin{array}{l}\text { Carcinoma ductal } \\
\text { Receptores }\end{array}$ & $91(82 \%)$ & $26(78,7 \%)$ \\
hormonales positivo & $87(78,3 \%)$ & $29(87,8 \%)$ \\
Gen HER 2 neu & $13(11,7 \%)$ & $2(6 \%)$ \\
\hline
\end{tabular}

Gen HER 2 neu: receptor 2 del factor de crecimiento epidérmico humano.

\section{DISCUSIÓN}

En este estudio se encontró una frecuencia similar de sobrepeso y obesidad en pacientes con cáncer de mama en un estudio realizado en Paraguay ${ }^{(16)}$, superior a otros estudios realizados en México y $\operatorname{Colombia}^{(17,18)}$ e inferior al estudio realizado por Riera Ledesma et al. en Cuba ${ }^{(19)}$. Se observa en los diferentes estudios la alta frecuencia de sobrepeso y obesidad en las mujeres con cáncer de mama, con variaciones de la frecuencia de un país a otro. La frecuencia similar encontrada de sobrepeso y obesidad en pacientes de cáncer de mama en pacientes del Hospital Nacional de Itauguá y el Instituto Nacional de Cáncer ${ }^{(16)}$ y este estudio sugiere la frecuencia aproximada de sobrepeso y obesidad presente en pacientes con cáncer de mama en el país.

La edad media de las pacientes estudiadas fue de 52 años, en la mayoría de los estudios realizados que evaluaron la frecuencia de sobrepeso y obesidad en pacientes con cáncer de mama se encontró en edad posmenopáusica, etapa de la vida en que se desarrolla con más frecuencia el cáncer de mama en las mujeres ${ }^{(10,13,19-21)}$.

La procedencia más frecuente de las pacientes con cáncer de mama fue de ciudades del departamento central, seguida por la ciudad de Asunción y en último lugar desde el interior del país. Tal distribución se debería en primer lugar a la densidad poblacional de la capital y el departamento central, y por otro lado en el Hospital de Clínicas, acuden pacientes de escasos recursos para el diagnóstico y tratamiento de todos tipos de enfermedades ${ }^{(22)}$.

En este estudio se encontró una frecuencia mayor de cáncer de mama del subtipo histológico ductal infiltrante similar al estudio realizado por Maffuz-Aziz et al. en México ${ }^{(23)}$ y de Bueno realizado en Perú( ${ }^{(2)}$. El subtipo histológico más frecuente de cáncer de mama en mujeres a nivel mundial es el ductal infiltrante ${ }^{(25,26)}$, en este estudio no difiere de los resultados de otros estudios.

La presencia de receptores hormonales se dio en el $80 \%$ de los casos de cáncer de mama, similar a los resultados de otros estudios ${ }^{(27,28)}$. La sobreexpresión del oncogén HER2neu fue del $10 \%$, Quevedo Gutiérrez et al. encontraron una frecuencia superior del oncogén HER2neu en pacientes con cáncer de mama en México ${ }^{(29)}$. El genotipo triple negativo se observó en el $16,6 \%$ de los casos de cáncer de mama, frecuencia mayor que en otros estudios ${ }^{(12,23)}$.

La limitación del estudio fue su diseño transversal y retrospectivo, que no permite demostrar con certeza el desarrollo de cáncer de mama en las mujeres con sobrepeso y obesidad, también no permite la generalización de los resultados en otra población. 
Este estudio podría ser útil para los médicos del Hospital de Clínicas y de otros hospitales del país para implementar medidas de prevención de cáncer de mama en mujeres, sobre todo posmenopáusicas disminuyendo los factores de riesgo cardiovasculares, específicamente el sobrepeso y la obesidad ${ }^{(30)}$ y permitió conocer la frecuencia del tipo histológico y característica inmunohistoquímica de las pacientes con cáncer de mama.

\section{Fuentes de financiamiento:}

Este trabajo ha sido financiado por los autores.

\section{Conflicto de interés}

Los autores declaran no tener conflictos de interés.

\section{REFERENCIAS BIBLIOGRÁFICAS}

1. Al Kibria GM. Prevalence and factors affecting underweight, overweight and obesity using Asian and World Health Organization cutoffs among adults in Nepal: Analysis of the Demographic and Health Survey 2016. Obes Res Clin Pract. abril de 2019;13(2):129-36.

2. Kontsevaya A, Shalnova S, Deev A, Breda J, Jewell J, Rakovac I, et al. Overweight and Obesity in the Russian Population: Prevalence in Adults and Association with Socioeconomic Parameters and Cardiovascular Risk Factors. Obes Facts. 2019; 12(1):10314.

3. Suárez-Carmona $W$, Sánchez-Oliver $A J$, González-Jurado JA, Suárez-Carmona W, Sánchez-Oliver AJ, González-Jurado JA. Fisiopatología de la obesidad: Perspectiva actual. Rev Chil Nutr. 2017; 44(3):226-33.

4. Avgerinos KI, Spyrou N, Mantzoros CS, Dalamaga $M$. Obesity and cancer risk: Emerging biological mechanisms and perspectives. Metabolism. marzo de 2019;92:121-35.

5. Bardou M, Barkun AN, Martel M. Obesity and colorectal cancer. Gut. junio de 2013;62(6):933-47.

6. Bandini M, Gandaglia G, Briganti A. Obesity and prostate cancer. Curr Opin Urol. septiembre de 2017;27(5):41521.

7. Picon-Ruiz M, Morata-Tarifa C, ValleGoffin JJ, Friedman ER, Slingerland JM. Obesity and adverse breast cancer risk and outcome: Mechanistic insights and strategies for intervention. CA Cancer J Clin. septiembre de 2017;67(5):37897/

8. Kang C, LeRoith D, Gallagher EJ. Diabetes, Obesity, and Breast Cancer. Endocrinology. 1 de noviembre de 2018; 159(11):3801-12.

9. Lee $K$, Kruper L, Dieli-Conwright CM, Mortimer JE. The Impact of Obesity on Breast Cancer Diagnosis and Treatment. Curr Oncol Rep. 27 de marzo de 2019; 21(5):41.
10. Fallone $F$, Deudon $R$, Muller C, Vaysse C. [Breast cancer, obesity and adipose tissue: a high-risk combination]. Med Sci MS. diciembre de 2018; 34(12): 1079-86.

11. Bhardwaj P, Au CC, Benito-Martin A, Ladumor $\mathrm{H}$, Oshchepkova S, Moges R, et al. Estrogens and breast cancer: Mechanisms involved in obesity-related development, growth and progression. J Steroid Biochem Mol Biol. mayo de 2019; 189: 161-70.

12. Chen L, Cook LS, Tang M-TC, Porter PL, Hill DA, Wiggins $C L$, et al. Body mass index and risk of luminal, HER2overexpressing, and triple negative breast cancer. Breast Cancer Res Treat. junio de 2016;157(3):545-54.

13. Nagrani R, Mhatre $S$, Rajaraman $P$, Soerjomataram I, Boffetta P, Gupta S, et al. Central obesity increases risk of breast cancer irrespective of menopausal and hormonal receptor status in women of South Asian Ethnicity. Eur J Cancer Oxf Engl 1990. octubre de 2016;66:153-61.

14. Kawai M, Malone KE, Tang M-TC, Li CI. Height, body mass index (BMI), BMI change, and the risk of estrogen receptor-positive, HER2-positive, and triple-negative breast cancer among women ages 20 to 44 years. Cancer. 15 de mayo de $2014 ; 120$ (10):1548-56.

15. Aboytes-Ríos B, García-Regalado $F$, Suárez-García D, Martínez-Garza S, Murillo-Ortiz B. Longitud de los telómeros y obesidad en mujeres con cáncer de mama. Rev Mex Mastol. 6 de octubre de 2020; 9(2-3):53-61.

16. León SJA, Cabral F, Escalada G, Cabral $L$, León MA, Gauna C. Factores de riesgo cardiovascular en pacientes con cáncer de mama ¿Existe correlación con las referencias internacionales? Rev Virtual Soc Paraguaya Med Interna. 31 de enero de 2020; 66-76.

17. Bertehelli-Cardona I, Ángeles-Casas $M$, Mejía-Miranda N, Martínez-Ángeles J. Obesidad en mujeres con cáncer de 
mama en un hospital general de zona de Hidalgo, México. Aten Fam. 21 de diciembre de 2017; 25(1):3-6.

18. Agudelo JMP, Avendaño BAC, Ruiz WAA, Pérez JFB. Perfil demográfico, clínico y farmacoterapéutico de pacientes con cáncer de mama en Caldas, Colombia. Rev Cuba Farm [Internet]. 26 de junio de 2018 [citado 19 de febrero de 2021]; 51(3). Disponible en: http://www.revfarmacia.sld.cu/index.ph $\mathrm{p} /$ far/article/view/147

19. Rivera Ledesma $E$, Fornaris Hernández A, Mariño Membribes ER, Alfonso Díaz K, Ledesma Santiago RM, Abreu Carter IC, et al. Factores de riesgo del cáncer de mama en un consultorio de la Atención Primaria de Salud. Rev Habanera Cienc Médicas. abril de 2019;18(2):308-22.

20. Martínez-Garza S, Suárez-García D, Murguía-Pérez M, Murillo-Ortiz B, González-García-Rojas EA. Cáncer de mama triple negativo y su relación con la obesidad. Rev Mex Mastol. 6 de octubre de $2020 ; 9(2-3): 38-43$.

21. Ayoub NM, Yaghan RJ, Abdo NM, Matalka II, Akhu-Zaheya LM, AlMohtaseb $\mathrm{AH}$. Impact of Obesity on Clinicopathologic Characteristics and Disease Prognosis in Pre- and Postmenopausal Breast Cancer Patients: A Retrospective Institutional Study. J Obes. 2019; 2019:3820759.

22. Gómez-Suárez R, Guanais FC. Serie sobre hospitalizaciones evitables y fortalecimiento de la atención primaria en salud: El caso de Paraguay I Publications [Internet]. 2012 [citado 23 de febrero de 2021]. Disponible en: https://publications.iadb.org/publication s/spanish/document/Serie-sobrehospitalizaciones-evitables-yfortalecimiento-de-la-atenci\%C3\%B3n-
primaria-en-salud-El-caso-de-

Paraguay.pdf

23. Maffuz-Aziz A, Labastida-Almendaro S, Espejo-Fonseca A, Rodríguez-Cuevas S. Características clinicopatológicas del cáncer de mama en una población de mujeres en México. Cir Cir. 1 de mayo de 2017; 85(3):201-7.

24. Bueno GAM. Características clínicas y pronósticas de los subtipos moleculares de cáncer de mama determinados por inmunohistoquímica. Arequipa, Perú. Rev Peru Med Exp Salud Pública. septiembre de 2017;34:472-7.

25. Khalil AI, Bendahhou K, Mestaghanmi $H$, Saile $R$, Benider A. [Breast cancer in Morocco: phenotypic profile of tumors]. Pan Afr Med J. 2016; 25:74.

26. Luangxay T, Virachith S, Hando K, Vilayvong $S$, Xaysomphet $P$, Arounlangsy $P$, et al. Subtypes of Breast Cancer in Lao P.D.R.: A Study in a Limited-Resource Setting. Asian Pac J Cancer Prev APJCP. 26 de febrero de 2019; 20(2):589-94.

27. Vasiliou SK, Diamandis EP. Androgen receptor: A promising therapeutic target in breast cancer. Crit Rev Clin Lab Sci. mayo de 2019;56(3):200-23.

28. Rodríguez-Silva CJ, González-Vela JL, Velázquez-Pacheco AA. Prevalencia de receptores androgénicos en el cáncer de mama. Gac Mex Oncol. 1 de mayo de 2015; 14(3):135-40.

29. Quevedo Gutiérrez KM, Landa Fernández AM, García Barrera V. HER$2 /$ neu en cáncer de mama localmente avanzado. Gac Mex Oncol. 1 de mayo de $2016 ; 15(3): 108-15$.

30. Ramírez $\mathrm{K}$, Acevedo $\mathrm{F}$, Herrera ME, Ibáñez C, Sánchez C. Actividad física y cáncer de mama: un tratamiento dirigido. Rev Médica Chile. enero de 2017;145(1):75-84. 